\title{
Hepatic stress associated with pathologies characterized by disturbed glucose production
}

\author{
Monika Gjorgjieva ${ }^{1-3, \#}$, Gilles Mithieux ${ }^{1-3}$ and Fabienne Rajas ${ }^{1-3, *}$ \\ 1 Institut National de la Santé et de la Recherche Médicale, U1213, Lyon, F-69008, France. \\ 2 Université de Lyon, Lyon, F-69008 France. \\ 3 Université Lyon I, Villeurbanne, F-69622 France. \\ \# Current address: Université de Genève, Geneva, 1211 Switzerland. \\ * Corresponding Author: \\ Dr. Fabienne Rajas, Ph.D, Inserm U1213, Université Lyon 1 Laennec, 7 rue Guillaume Paradin, 69372 Lyon cedex 08 France; Tel: +33 4 \\ 787710 28; Fax: +33 4787787 62; E-mail: fabienne.rajas@univ-lyon1.fr
}

\begin{abstract}
The liver is an organ with many facets, including a role in energy production and metabolic balance, detoxification and extraordinary capacity of regeneration. Hepatic glucose production plays a crucial role in the maintenance of normal glucose levels in the organism i.e. between 0.7 to $1.1 \mathrm{~g} / \mathrm{l}$. The loss of this function leads to a rare genetic metabolic disease named glycogen storage disease type I (GSDI), characterized by severe hypoglycemia during short fasts. On the contrary, type 2 diabetes is characterized by chronic hyperglycemia, partly due to an overproduction of glucose by the liver. Indeed, diabetes is characterized by increased uptake/production of glucose by hepatocytes, leading to the activation of de novo lipogenesis and the development of a non-alcoholic fatty liver disease. In GSDI, the accumulation of glucose- 6 phosphate, which cannot be hydrolyzed into glucose, leads to an increase of glycogen stores and the development of hepatic steatosis. Thus, in these pathologies, hepatocytes are subjected to cellular stress mainly induced by glucotoxicity and lipotoxicity. In this review, we have compared hepatic cellular stress induced in type 2 diabetes and GSDI, especially oxidative stress, autophagy deregulation, and ER-stress. In addition, both GSDI and diabetic patients are prone to the development of hepatocellular adenomas (HCA) that occur on a fatty liver in the absence of cirrhosis. These HCA can further acquire malignant traits and transform into hepatocellular carcinoma. This process of tumorigenesis highlights the importance of an optimal metabolic control in both GSDI and diabetic patients in order to prevent, or at least to restrain, tumorigenic activity during disturbed glucose metabolism pathologies.
\end{abstract}

doi: $10.15698 /$ cst2019.03.179

Received originally: 10.09 .2018

in revised form: 26.11.2018,

Accepted 10.12.2018,

Published 28.01.2019.

Keywords: glucose-6 phosphate, diabetes, glycogen storage disease type $I$, glucotoxicity, lipotoxicity, NAFLD, hepatic tumors.

\footnotetext{
Abbreviations:

AGE-advanced glycation end-product,

ChREBP - carbohydrate response element binding protein,

ECM - extracellular matrix,

$E G P$ - endogenous glucose production,

G6P-glucose-6-phosphate,

G6Pase-glucose-6-phosphatase,

GNG - gluconeogenesis,

GSDI - glycogen storage disease type I,

HCA - hepatocellular adenoma,

HCC - hepatocellular carcinoma,

iNOS - inducible nitric oxide synthase,

NAFLD - non-alcoholic fatty liver disease,

NEFA - non-esterified fatty acid,

$P P P$ - pentose phosphate pathway,

$R O S$ - reactive oxygen species,

SREBP-1c - sterole regulatory element binding protein-1c,

$T G$ - triglyceride,

TNFa - tumor necrosis factor alpha,

$V L D L$ - very low density lipoprotein.
}

\section{LIVER FUNCTION IN GLUCOSE PRODUCTION AND NORMOGLYCEMIA}

Maintaining normal glucose levels in the organism i.e. between 0.7 to $1.1 \mathrm{~g} / \mathrm{L}$, is a complex task, involving a multiorgan crosstalk responsible for metabolic homeostasis. This molecular machinery is crucial for the normal func- tioning of the body, given that glucose is considered one of the main metabolites ensuring energy production in the cells. "Energy source" has always been the main function attributed to glucose. However, glucose plays other essential roles in the cell, such as providing carbon skeletons on which all other specialized biochemical pathways ultimate- 
ly depend [1]. Indeed, glucose can be a limiting factor in cell proliferation not only by its energetic role, but above all by providing carbons for nucleotide synthesis via the pentose phosphate pathway (PPP), required for DNA replication. Thus, the various roles of glucose impose a strict regulation of the concentration of this metabolite in the organism, while aberrations in the maintenance of normoglycemia can induce deleterious phenotypes and pathological states.

\section{Glucose production during fasting periods}

During fasting periods, glucose is consumed by all organs, resulting in a decrease in glucose levels. Normoglycemia is maintained by endogenous glucose production (EGP), a process occurring in the liver, as well as in the kidneys and in the intestine [1]. EGP is activated by glucagon or counter-regulatory hormones such as epinephrine or norepinephrine. Hepatic EGP relies on two different pathways: glycogenolysis and gluconeogenesis (GNG). Renal and intestinal EGPs rely only on GNG. Short-term fasts induce the hepatic glycogenolysis pathway, entailing a degradation of glycogen into glucose- 6 phosphate (G6P), which is further hydrolyzed into glucose. Once hepatic glycogen stores are depleted during prolonged fasts, hepatocytes activate GNG and thus use amino acids, lactate, pyruvate and glycerol as substrates in order to synthetize glucose de novo. Therefore, glycogenolysis and GNG use different substrates for glucose production, yet share the last reaction - the hydrolysis of G6P into free glucose and inorganic phosphate by glucose- 6 phosphatase (G6Pase). While hepatic glucose production is a widely known process, renal and intestinal GNG has been often neglected, even though they contribute immensely to glycaemia homeostasis during long-term fasting. Indeed, during $24 \mathrm{~h}-48 \mathrm{~h}$ fasting, kidney and intestine can be responsible for up to $50 \%$ and $20 \%$ of glucose production, respectively, as shown in rodents, which corroborates comparable results in humans [1-6]. In addition, intestinal GNG plays a central regulatory role in energy homeostasis in the post-absorptive state. By delivering glucose directly in the portal vein, intestinal GNG induces a gut-brain glucose signal that positively controls different metabolic functions, such as food intake and insulin sensitivity [1]. Failure to activate these physiological pathways due to metabolite imbalance or improper signalization caused by genetic, nutritional or environmental reasons can result in hypoglycemia.

\section{Glucose storage during postprandial/post-absorptive periods}

After the ingestion of a meal, different nutrients such as sugars, lipids and proteins are digested and absorbed. The absorption of glucose raises the circulating concentration of this metabolite in the blood stream. While glucose is essential for the normal functioning of the organism, excessive amounts can induce glucotoxicity $[7,8]$. In order to prevent glucotoxicity and to form glucose stores needed during fasting, glucose is captured by the liver and the peripheral tissues and stored under the form of glycogen and lipids. This process is orchestrated by insulin, which is known to activate glycogen synthesis, lipogenesis, as well as protein synthesis. Failure to restore normoglycemia after meals entails hyperglycemia and diabetes.

In this review, we will focus on the cellular stress induced in the liver under two different pathophysiological states linked to deregulation in EGP, inducing either chronic hyperglycemia (Type 2 diabetes) or hypoglycemia (Glycogen Storage Disease type I-GSDI). In both cases, this deregulation leads to the development of fatty liver disease and, in some patients, it can lead to hepatic tumor development (Figure 1).

\section{DEREGULATION IN ENDOGENOUS GLUCOSE PRODUC- TION AND FATTY LIVER}

Type 2 diabetes is the most common condition characterized by chronic hyperglycemia. With the expansion of the western civilization lifestyle, the incidence of this disease, along with obesity, has dramatically risen worldwide [9]. Type 2 diabetes is characterized by insulin resistance of the tissues capable of capturing glucose and/or the lack of insulin production due to $\beta$-cell function decline [10]. As mentioned, chronically elevated glucose levels in the bloodstream can induce glucotoxicity. Most studies in diabetes-associated glucotoxicity address this phenomenon in the $\beta$-cells, as a negative retroactive system amplifying insulin secretion dysfunction. However, in the liver, dissociating the exact mechanisms behind cell stress induced by glucose toxicity or by lipid toxicity, such as in diabetic and/or obese patients is very difficult. Glucose and lipid metabolisms are tightly linked, due to the interchange of common metabolites. Thus, diabetes, characterized by glucose metabolism dysfunction, is linked to liver disease, more precisely to non-alcoholic fatty liver disease (NAFLD) $[11,12]$. Indeed, up to $70 \%$ of diabetic patients may present NAFLD [11, 12]. Even if glucose uptake is impaired in obese/diabetic mice [13], elevated levels of blood glucose induce an increased metabolic flux downstream of G6P and a subsequent activation of de novo lipogenesis (Figure 2) [14]. Insulin and glucose can both induce hepatic lipogenesis. Interestingly, insulin-mediated lipogenesis is activated via sterol regulatory element-binding protein-1c (SREBP1c), while glucose-mediated lipogenesis is activated via carbohydrate response element binding protein (ChREBP) $[15,16]$. More precisely, glucose metabolites such as G6P or xylulose 5-phosphate were suggested to directly activate ChREBP [15]. SREBP-1c and ChREBP are key transcription factors in lipogenesis [17]. Paradoxically, even in insulin-resistant states, this hormone still manages to activate hepatic lipogenesis via SREBP-1c. De novo lipogenesis is not the only process contributing to fatty liver. Indeed, in crease in hepatic lipid storage also results from diet, elevated non-esterified fatty acids (NEFA) due to a decreased inhibition of adipose tissue lipolysis, reduced hepatic lipid oxidation, as well as reduced lipid export in the form of very low density lipoprotein (VLDL) [18]. Finally, hepatic lipids tend to further accentuate insulin resistance by interfering with insulin signaling, thus enclosing a vicious cycle. 


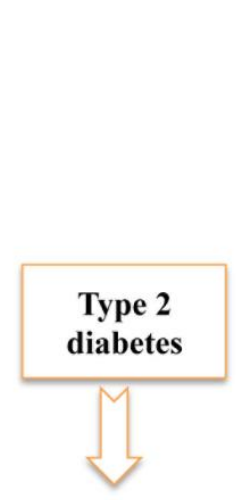

High

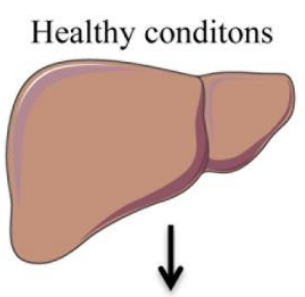

Hepatic glucose production

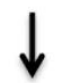

Blood glucose [0.7-1.1 g/l] glucose production

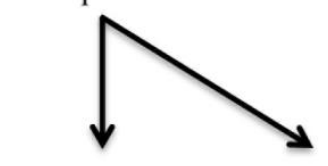

Hyperglycemia

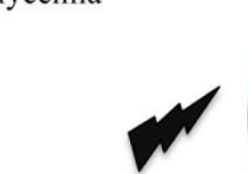

Glucotoxicity Lipotoxicity

\section{NAFLD}

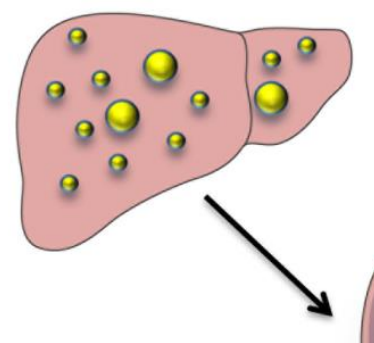

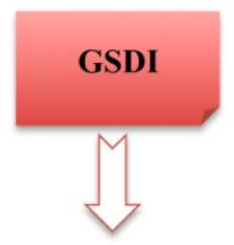

Absence of glucose production

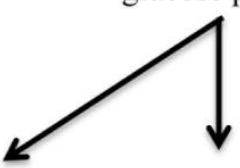

Hypoglycemia

FIGURE 1: Deregulation of endogenous glucose production leads to hepatic complications. In normal physiological conditions, the liver maintains glucose homeostasis in the blood by releasing free glucose during fasts and by up-taking and storing excessive glucose during post-prandial periods. In type 2 diabetes, hepatic production and uptake of glucose is deregulated, resulting in hyperglycemia. In Glycogen Storage Disease type I (GSDI), glucose production in completely abolished, resulting in hypoglycemia. Strikingly, both of these pathologies are characterized by nonalcoholic fatty liver disease (NAFLD) development that can lead to hepatocellular carcinoma in some cases.

$\mathrm{HCA} / \mathrm{HCC}$

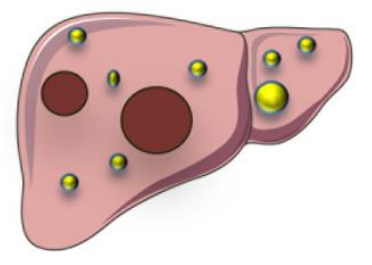

While hyperglycemia is widely spread and classified as an epidemic, chronic hypoglycemia (plasma glucose level lower than $0.55 \mathrm{~g} / \mathrm{L}$ ) is not a very common condition, although acute hypoglycemic episodes are often experienced in diabetic patients undergoing unstable therapy or receiving excessive amounts of insulin. Interestingly, patients with GSDI, a rare genetic disease $(1 / 100,000$ live births) suffer from chronic hypoglycemia during short fasting periods [19]. Indeed, as opposed to type 2 diabetes, this pathology is characterized by an absence of EGP due to a deficiency in G6Pase (Figure 1 and 2) [19-21]. This leads to the accumulation of G6P in hepatocytes. As in diabetic patients (see above), increased G6P activates glycogen synthesis and de novo lipogenesis, leading to hepatomegaly and severe steatosis induced by strong glycogen and lipid accumulation, respectively (Figure $\mathbf{1}$ and 2), associated with hypercholesterolemia and hypertriglyceridemia [ 22 23]. In GSDI human livers, de novo lipogenesis and cholesterol synthesis were found to be increased 40-fold and 7fold, respectively [24]. Furthermore, conversion of VLDL into intermediate density lipoproteins is delayed. Lipid vesicles are present in abundance in GSDI livers, mainly in the periportal zone, which corresponds to the location of the highest expression of G6Pase in the liver [25]. In addi- tion, high G6P levels induce glycolysis and PPP, leading to lactic acidosis and hyperuricemia, respectively [26-28].

Before developing the molecular events in regards to cell stress in diabetic and GSDI hepatocytes, it is noteworthy that both of these pathologies are characterized by an increase of the metabolic flux downstream of G6P [29]. This elevation leads to a deep metabolic reprogramming, which induces cell stress in the liver of both diabetic and GSDI patients [26].

\section{OXIDATIVE STRESS IN THE CONTEXT OF} HYPERGLYCEMIA AND HEPATIC STEATOSIS

The glucotoxic and lipotoxic effects in the liver of diabetic subjects have many facets. Oxidative stress is one of the mechanisms behind this toxicity in the liver [30-33], but it presents as a multi-organ pathology that can also be responsible for $\beta$-cell loss-of-function, vascular complications and strokes, neuropathy, retinopathy and nephropathy in diabetes [34].

The mechanisms of G6P-derived cell stress include increased polyol pathway flux, increased intracellular formation of advanced glycation end-products (AGEs), activation of protein kinase $C(P K C)$, increased hexosamine pathway or overproduction of superoxides by the mitochondrial electron transport chain [35-38]. The polyol 
pathway leads to the conversion of glucose to sorbitol and further to fructose, thus reducing the availability of cofactors such as NADPH needed for the glutathione peroxidase-glutathione reductase system, rendering the cell more vulnerable to reactive oxygen species (ROS), and therefore reinforcing oxidative stress $[39,40]$. Furthermore, during chronic hyperglycemia, glucose can be autooxidized and form covalent adducts with the plasma proteins through a non-enzymatic process known as glycation, leading to the formation of AGEs [41-43]. Glycation of proteins interferes with their normal functions by disrupting molecular conformation, altering enzymatic activity and interfering with receptors' function. AGEs interact with receptors for AGEs (RAGE) to alter intracellular signaling, gene expression, release of pro-inflammatory molecules and free radicals. Finally, the increase in the hexosamine pathway flux is a complex process involving the usage of glucose to produce glucosamine-6-phosphate and subse- quently UDP-N-acetylglucosamine (UDP-GlcNAc), resulting in pro-fibrotic signalization, characterized by the induction of transforming growth factor $\beta 1$ (TGF- $\beta 1$ ) and plasminogen activator inhibitor-1 (PAI1) expression $[44,45]$.

Oxidative stress is due to an increased amount of ROS in the cell that can result from decreased antioxidant activity and/or increased ROS production. ROS notably include superoxide anion $\left(\mathrm{O}_{2}^{-}\right)$, hydrogen peroxide $\left(\mathrm{H}_{2} \mathrm{O}_{2}\right)$ and hydroxyl radical ( $\left.\mathrm{HO}^{-}\right)$. ROS production is mainly attributed to the mitochondrial complexes in the electron transport chain, responsible for the oxidative phosphorylation, as well as NADPH oxidases, xanthine oxidase, nitric oxide synthase. The production of these highly unstable molecules is a physiological process that is tightly regulated by antioxidant activity, in order to prevent the negative cellular outcome of ROS. However, in pathological conditions, antioxidant activity can be exceeded or insufficient to restore the physiological concentration of ROS and thus lead to oxida-

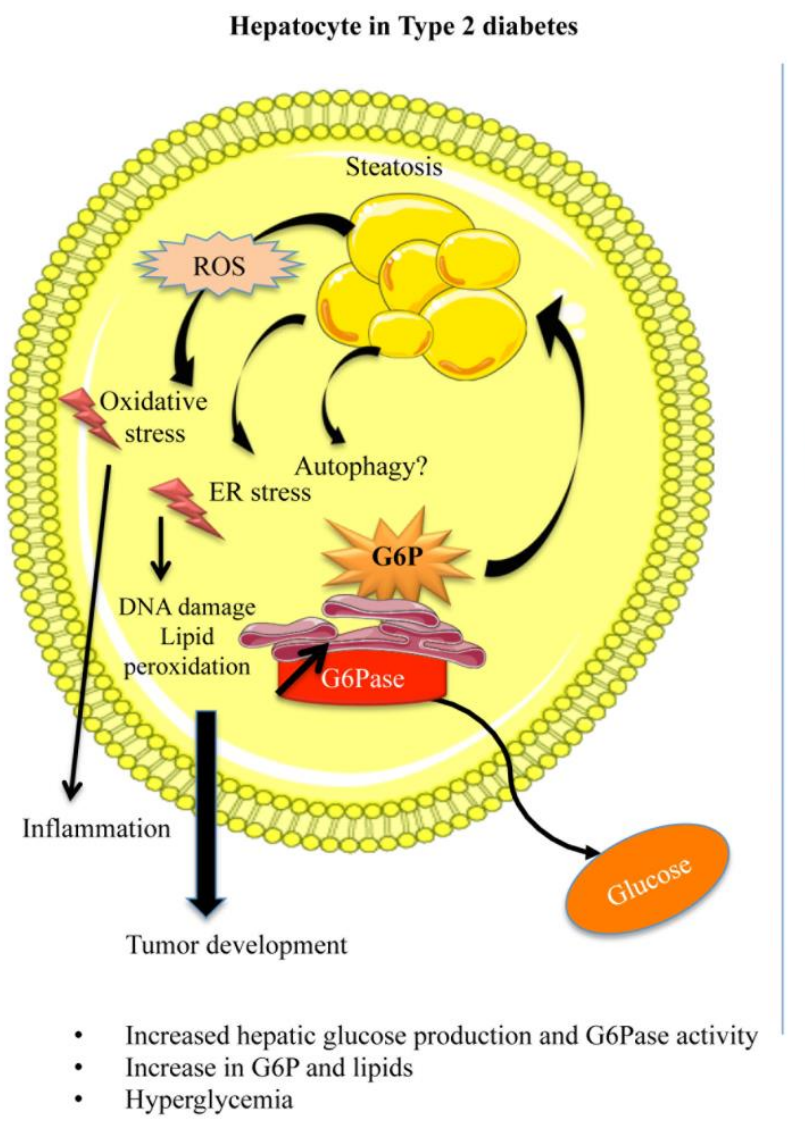

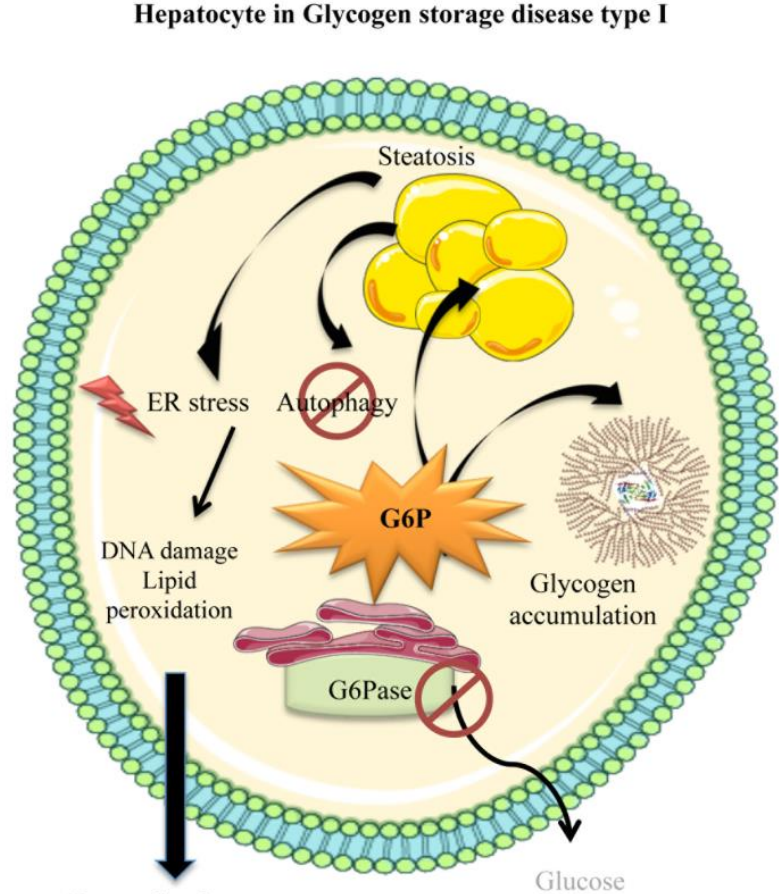

Tumor development

- Absence of glucose production and loss of G6Pase activity

- Increase in G6P and lipids

- Hypoglycemia

FIGURE 2: Dysfunction of hepatocyte metabolism in type 2 diabetes and GSDI leads to cell stress. Type 2 diabetes is associated with hyperglycemia partially due to an overproduction of glucose by the liver, since G6Pase activity is increased, whereas GSDI is associated with hypoglycemia due to the absence of G6Pase activity. However, these two diseases share similar hepatic metabolism leading to the development of fatty liver. In type 2 diabetes, hyperglycemia leads to an increase of the metabolic flux downstream of G6P, whereas in GSDI, the absence of G6Pase activity is responsible for G6P accumulation. In both diseases, this results in an activation of de novo lipogenesis. In addition, GSDI is characterized by glycogen accumulation. These metabolic perturbations are responsible for cell stress such as ER stress. Even though mitochondrial dysfunctions were shown in both diseases, increased ROS production and oxidative stress has only been observed in diabetes, but not in GSDI. In GSDI, autophagy is also clearly decreased, but this is still controversial in diabetes. In both diabetes and GSDI, cell stress could cause DNA and protein damages, lipid peroxidation and finally, the development of hepatic tumors. 
tive stress. Indeed, during cell stress and/or mitochondrial insult, mitochondrial complex I and III mainly contribute to oxidative stress by producing $\mathrm{O}_{2}^{-}$[46]. In addition, membrane-bound NADPH oxidases can be activated by inflammatory signals and can also lead to the production of $\mathrm{O}_{2}^{-}$. Diabetic animals present decreased hepatic activity of antioxidant enzymes such as catalase and superoxide dismutase 1 , leading to increased ROS and hydroperoxides $[47,48]$. The increase in ROS levels can damage lipids, proteins, DNA, RNA and can affect the functioning of various organelles in the cell, such as the mitochondria or the endoplasmic reticulum (ER), thus leading to a pathological state in the hepatocytes $[32,48]$. For example, lipid peroxidation is a process where ROS can interact with the lipid's electrons and thus alter its structure and characteristics, leading to important disturbances in the bi-layer lipid membranes of the cell, which can have strong effects on cell homeostasis and signalization, as well as survival. Taking into consideration that type 2 diabetes is associated with hepatic lipid accumulation, oxidative stress represents a major issue in liver function in these patients. Furthermore, ROS can easily target the reduction state of sulphurcontaining amino acids, cysteine (Cys) and methionine (Met) with great impact on the protein structure and/or enzymatic activity. Hydroxylation and carboxylation of proteins mediated by ROS can also occur during oxidative stress. Moreover, the quantification of the latter is often used to assess the extent of oxidative damage in the cells [49]. Protein damage and aggregation can easily impact the functioning of the ER and thus induce ER stress. In regards to DNA, ROS can induce structural modifications of the bases, inter- and intra-strand crosslinks, induce strand breaks and promote DNA-protein crosslinks [50]. Indeed, oxidative stress-induced DNA damage has been documented in diabetes [51]. Last, ROS overproduction leads to depletion in adenosine triphosphate (ATP) and nicotinamide dinucleotide (NAD), which directly affects energy homeostasis in the cell [52].

\section{ROS and lipids}

While the lipid storage capacity of adipose tissues is much higher than other tissues, the liver is able to store large amount of lipids in form of triglyceride (TG), diacylglycerol and cholesterol esters [53]. Hepatic lipids accumulate in cytoplasmic lipid droplets and form highly dynamic organelles also containing proteins, such as perilipin [54, 55]. Many studies suggest that TG, even in high amounts in the liver, when stored in lipid vesicles, are relatively inert, nonreactive and thus rather inoffensive. While the storage of NEFA in form of TG represents a protective mechanism [56], their excessive accumulation and lipid droplet enlargement may lead to cell damage $[57,58]$. Indeed, NEFA spillover or the inability to store further NEFA in TG due to lack of capacity to further enlarge lipid droplets and the accumulation of specific lipotoxic compounds could lead to increased oxidative stress. Thus, lipids like ceramides, diacylglycerol and phosphatidic acid were shown to contribute strongly to insulin resistance and therefore, to amplify the diabetic phenotype $[59,60]$. Furthermore, free choles- terol and fatty acids are attracting more and more attention as the designated cell injury drivers in hepatic lipidrelated pathologies, due to their important reactivity with other components of the cell. First, increased presence of fatty acids in the liver can induce the production of free radicals. Indeed, the oxidation of fatty acids in the mitochondria, peroxisomes and microsomes, mediated in part by cytochrome P450 (CYP2E1), CYP4A10, and CYP4A14, results in an increase in ROS $[16,61]$. Conversely, ROS are responsible for increased lipid peroxidation. Trans-4hydroxy-2-nonenal (HNE) and malondialdehyde (MDA) are some of the most studied lipid peroxidation products that can be highly toxic in the cells. These molecules can interact with DNA and form etheno-DNA adducts, leading to carcinogenesis [62]. Moreover, aberrant lipid metabolism in diabetic/obese patients can lead to an induction of ER stress [63]. Indeed, hepatic lipid metabolism deregulation was shown to alter the composition of the phospholipids within the ER and thus perturb protein synthesis [64]. Moreover, protein aggregation in lipotoxic conditions can also induce this process. While ER stress can be induced by lipid misbalance, this process has an important pivotal role on lipid metabolism as a whole. For example, forced expression of $\mathrm{BiP}$, a key negative regulator of the ER stress response, was shown to protect against hepatic steatosis by inhibiting SREBP1c mediated lipogenesis [65]. Indeed, all of the components of the ER stress response, which includes the IRE1a/XBP1 axis, the PERK/ATF4 axis and ATF6 have all been shown to promote lipogenesis when activated, mainly by promoting SREBP1c expression, but also directly increasing the expression of lipogenic genes such as FAS, ACC and SCD1 [65]. As expected, attenuating ER stress in obese rodents decreases steatosis and improves insulin sensitivity [66].

\section{Oxidative stress and inflammation}

Oxidative stress results in an increase in apoptosis of hepatocytes and a subsequent release of inflammatory cytokines, attracting infiltration of the liver by inflammation-mediated leukocytes [67]. Pro-inflammatory mediators involved in hyperglycemic liver damage include interleukins such as IL-1 and IL-6, nuclear factor (NF-kB), mitogen-activated protein kinase (MAPK), TGF- $\beta 1$, poly ADPribose polymerase (PARP) and tumor necrosis factor- $\alpha$ (TNF $\alpha$ ) [31, 68-70]. Indeed, diabetic rat models confirmed that in the liver, the induction of TNF $\alpha$ results in increased levels of NF-kB and JNK signaling, characterized by further induction of inducible nitric oxide synthase (iNOS) and consequent increase in nitric oxide production, as well as increased apoptosis rates [71]. Specific inhibition of TNFa resulted in a decrease in the before-mentioned pathways, as well as decreased apoptosis [71]. Treatment with antioxidants such as Tempol also prevented lipid peroxidation and apoptosis induced by TNF $\alpha$ and iNOS and the subsequent oxidative stress [72]. On the other hand, increased circulating levels of II-6 were found to be responsible for the activation of another inflammatory pathway (STAT3dependent) in the liver $[73,74]$. While II-6 is a mitogen 
required for efficient regeneration of the liver, chronic II-6 activation could have a strong impact on the hepatocytes and can be involved in the development of a pathological state. Strikingly, other studies have shown that II-6 can possibly have an anti-inflammatory role in diabetes, confirming that the molecular signalization in diabetes is quite complex [75].

The production of these inflammatory factors is due to various cell types infiltrating the liver, such as neutrophils, macrophages and T-lymphocytes, depending on the stage and condition of the hepatic pathology [76]. Furthermore, resident cells in the liver, such as Kupffer cells (macrophages) and even hepatocytes can also play a major role in inflammation mediation. Interestingly, inflammation seems to play a key role in promoting the progression of NAFLD to steatohepatitis and further to cirrhosis and cancer. For example, significant infiltration of T cells is detected in patients with NAFLD and correlates with the disease severity, suggesting that $T$ cells promote the progression of NAFLD [77]. In addition, it was shown that metabolic changes linked to NAFLD promote a selective inhibition of $\mathrm{CD}^{+} \mathrm{T}$ lymphocytes infiltration, while $\mathrm{CD}^{+}$lymphocytes were unaffected, leading to an acceleration of hepatic carcinogenesis [78]. Thus, the activation of inflammatory cells in the liver leads to hepatocyte injury and liver fibrosis by producing ROS and inflammatory mediators (as described above). However, inflammation can also have beneficial effects by stimulating removal of dead cells and liver regeneration. Thus, inflammatory cells and mediators in the liver could have multifaceted functions in the liver by promoting pathogenesis progression of NAFLD or protecting hepatocytes against apoptosis, as described by Gao and Tsukamoto (2016) [76]. Finally, inflammation can also have a direct role on insulin sensitivity. Indeed, ROS can inhibit insulin signaling by inducing Insulin Receptor Substrate (IRS) degradation in peripheral tissues, thus leading to insulin desensitization [79]. Similarly, TNF $\alpha$ was also shown to induce insulin resistance.

\section{HEPATIC OXIDATIVE STRESS AND INFLAMMATORY STATUS IN GSDI}

While deciphering the clinical aspects of GSDI is a highly important task, this pathology has much to offer to basic research as well. Indeed, GSDI is characterized by chronic hypoglycemia in the absence of treatment, yet in the hepatocytes these patients present extreme metabolic features comparable to those observed in diabetes. Paradoxically, GSDI patients present increased risk of insulin resistance even though they suffer from hypoglycemia, when they are not under optimal nutritional care [80]. In order to study GSDI, several mouse models have been developed, including total deletion and liver-specific deletion of the gene encoding the catalytic subunit of the G6Pase $(G 6 p c)$ [81, 82]. Total deletion mouse models present severe hypoglycemia especially in the absence of oral or injected glucose, leading to premature death in these mice after weaning. However, liver-specific G6pc-deficient (L.G6pc-/-) mice are viable, rendering this model particular- ly well suited for long-term studies. Indeed, L.G6pc-/- mice can produce glucose from their kidneys and intestine during fasting [83]. Both of these models develop the hepatic pathology observed in GSDI patients, including hepatomegaly and hepatic steatosis, associated with hypercholesterolemia and hypertriglyceridemia [81, 82].

\section{Mitochondrial dysfunction and ROS}

Mouse models have generated interesting data in regards to cell stress associated with GSDI. As previously mentioned, G6Pase deficiency leads to hepatocyte metabolism characterized by the activation of glycolysis, de novo lipogenesis, PPP and glycogen synthesis [22, 23]. Interestingly, hepatic mitochondrial dysfunction was reported, along with a striking decrease in basal respiration, ATP turnover, maximal respiration, and spare mitochondrial capacity [84]. The structure of mitochondria was abnormal and the mitochondrial content was also decreased, probably due to decreased biogenesis. Another study confirmed this result in L.G6pc-/- mice, showing that lipid-mediated Sirtuin1 (SirT1) down-regulation entails a decrease in peroxisome proliferator-activated receptor- $\gamma$ coactivator $1 \alpha$ (PGC- $1 \alpha)$, and thus alters mitochondrial integrity, biogenesis, and function in GSDI hepatocytes [85]. The mitochondrial apoptosis pathway is also activated [84]. Indeed, an increase in cytochrome c release, as well as activation of caspases 9 and 3 were reported in G6pc-knock down cells. Finally, mitochondrial dysfunction was linked to insulin resistance [80]. Despite this pathological mitochondrial phenotype, ROS levels were not increased in the cells, leaving room to speculate that oxidative stress might not be present in the case of GSDI. Furthermore, increased circulating levels of antioxidants reported in GSDI patients could contribute to the protection against oxidative stress [86]. Elevated circulating antioxidants could also protect GSDI patients against atherosclerosis, despite hyperlipidemia $[87,88]$. It is noteworthy that hyperuricemia, albeit a pathological state, can also provide antioxidant defense, since plasma uric acid is also a potent low-molecular-weight antioxidant. However, within the cell, uric acid can have pro-oxidative roles as well, by forming radicals with other oxidants, rendering the effect of this metabolite in GSDI complex [89].

Since in GSDI the capacity of G6P storage under the form of glycogen is chronically exceeded, G6P activates de novo lipogenesis and leads to hepatic steatosis. Hepatic steatosis in GSDI is also enhanced by a decrease in lipid $\beta$-oxidation. This catabolic pathway was shown to be down-regulated in the liver of L.G6pc-/- mice [90], with a concomitant down-regulation of the main activator of $\beta$-oxidation, PPAR $\alpha$. It has been suggested that the production of malonyl CoA by acetyl CoA carboxylase (ACC) during lipogenesis could further contribute to the decrease in $\beta$-oxidation in GSDI livers. Interestingly, a reactivation of PPAR $\alpha$ in the liver of L.G6pc-/- mice via its agonist, fenofibrate, resulted in a normalization of the hepatic TG content and thus a complete disappearance of hepatic steatosis in these mice [91]. Strikingly, fenofibrate treatment resulted in a normalization of the glycogen content in 
L.G6pc-/- mice as well. Finally, a decreased activity of AMPactivated protein kinase (AMPK) in GSDI hepatocytes might also contribute to impaired fatty acid oxidation and increased fatty acid and cholesterol synthesis [92]. AMPK regulates these processes by decreasing malonyl CoA production via ACC inhibition and via the control of SREBP1 and ChREBP activities. A decrease in $\beta$-oxidation could thus contribute to the absence of increased ROS in GSDI livers.

\section{Autophagy}

Interestingly, altered lipid metabolism affects autophagy in GSDI hepatocytes (Figure 2). Indeed, lipid accumulation due to increased ChREBP and decreased PPAR $\alpha$ results in a decreased SIRT1/FOXO signaling and thus in the absence of autophagy activation [93]. Since SIRT1 is down-regulated during lipogenesis, it entails a vicious cycle between lipid accumulation and autophagy in GSDI. Indeed, SIRT1 is blocked due to lipid synthesis, which subsequently blocks autophagy and leads to further lipid accumulation. Besides lipids, other metabolites, proteins and even dysfunctional organelles remain non-recycled and lead to cell stress or even contribute to malignancy. In accordance, the reactivation of autophagy pathway in GSDI resulted in an increase in lipid degradation, associated with an improved hepatic histology [92]. It is noteworthy that in L.G6pc-/mice autophagy was found activated in the hepatic tumors, compared to the surrounding non-tumoral tissue [27]. As observed in many cancer types, this activation of autophagy in GSDI tumors could facilitate their progression by providing malignant cells with substrates for rapid proliferation, as well as a protective role against cell necrosis and inflammation [94].

Autophagy is a process that is considered as regulated by mTOR, AMPK and SIRT1 [95]. In the case of GSDI, autophagy was shown to be independent from mTOR signaling, since mTOR inhibition using Temsirolimus did not lead to autophagy activation in L.G6pc-/- mice [27]. As mentioned earlier, AMPK is strongly down-regulated in GSDI livers due to their energetic state, leaving only SIRT1 as a master regulator.

As observed in GSDI, NAFLD and diabetes can also be characterized by a decrease in autophagy. However, contradictory results showing ER stress-mediated induction of autophagy in obesity, rather than a decrease, have been reported as well. This highlighted that the levels of insulin resistance, steatosis and the overall state of the hepatocytes have a role to play in the outcome of this process [96]. Interestingly, one of the metabolites by which autophagy decrease in obesity can be mediated is nitric oxide. Indeed, obesity promotes S-nitrosylation of lysosomal proteins in the liver, thereby impairing lysosomal enzyme activities, and further facilitating hepatic steatosis and insulin resistance [97]. The canonical pathways regulating autophagy are involved in autophagy repression as well. Indeed, over-nutrition provides increased availability of amino acids and glucose in obesity, which can constitutively activate mTOR and inhibit AMPK, resulting in autophagy repression
[98]. Lipids, as in GSDI, can also contribute to autophagy inhibition. However, as lipids constitute a great family of molecules with different attributes, their differential effects on autophagy can vary greatly. For example, oleic acid was shown to induce autophagy, whereas palmitic acid suppresses this process [99]. Thus while in GSDI autophagy was proven to be systematically repressed, this is not always true in diabetes and obesity, probably due to the variability in the etiology, the staging of the pathology and the variable environment in diabetic and/or obese patients.

\section{Inflammatory status in GSDI livers}

Despite the important levels of accumulated glycogen and lipids in the liver, as well as the important metabolic imbalance, GSDI patients present low-grade hepatic inflammation $[19,100]$. However, a significant elevation of serum II-8 levels was reported in patients bearing tumors, positively correlating with neutrophilia and hepatic neutrophil infiltration [100]. Hepatic transaminase (AST/ALT) levels are also usually normal, especially in patients with optimal metabolic control and patients not bearing hepatic tumors [100].

The absence of oxidative stress and inflammatory responses in the case of GSDI might be the reason as to why hepatic fibrosis is not associated with GSDI [19]. Indeed, GSDI patients and related animal models do not present fibrosis in the liver, contrarily to other types of glycogen storage diseases. Consequently, these patients do not develop cirrhosis. However, they present a highly elevated risk of hepatic malignancy, characterized by a specific tumorigenic process described below.

\section{HEPATIC CARCIOGENESIS IN DIABETES AND GSDI}

Nowadays it is becoming more and more evident that diabetes is associated with chronic liver disease [101], leading to an important risk of hepatocellular carcinoma (HCC) development [78-80]. Interestingly, it was suggested that similar pathways are activated in both diabetes and hepatocellular cancer [102, 105]. For example, the Insulin/Insulin Growth factor 1 (IGF1) signalization pathway and the subsequent activation of mTOR are increased in both cases. In hyperinsulinemic conditions, insulin exerts a mitogenic role, rather than a metabolic role, which is highly beneficial for HCC progression [106]. Furthermore, the aforementioned inflammatory mediators such as TNF $\alpha$ and II-6 can also contribute to hepatic cancer development. Last, taking into account that cancer cells are often highly dependent on free glucose fueling the Warburg effect, chronic hyperglycemia is ideal for their rapid progression. In addition, hyperglycemia was shown to induce nuclear $\beta$-catenin accumulation in cancer cells, which could be yet another trigger of tumorigenesis in diabetes [107].

Interestingly, lipid-mediated expression of TNF $\alpha$ and oxidative stress are responsible not only for cell injury, inflammation, necrosis, but also for activation of stellate cells inducing fibrosis [108, 109]. Indeed, in the livers exposed to chronic injury, stellate cells promote the devel- 
opment of fibrosis through excessive extracellular matrix (ECM) production and reduced ECM degradation [110]. Reduced adiponectin levels can also potentiate the fibrogenic process [111]. Fibrosis is a common end point to chronic inflammation in insulin-resistant livers and it can be further stimulated by Kupffer cells, the resident hepatic macrophages. The formation of Mallory-Denk bodies, composed of misfolded intermediate filaments, ubiquitin, heat shock proteins, and p62, can be observed during fibrogenesis [112]. Hepatic fibrosis can further evolve to cirrhosis and HCC development (Figure 3). Linking cirrhosis and diabetes is very complex since cirrhosis itself is linked to insulin resistance [113]. Indeed, around $30 \%$ of patients with hepatic cirrhosis present diabetes, while cirrhosis is not necessarily induced by obesity / diabetes [114].
It is noteworthy that an important fraction of obese patients develop HCC in the absence of liver cirrhosis as well $[115,116]$. Indeed, around $54 \%$ of NAFLD patients diagnosed with HCC were not classified as cirrhotic, as opposed to only $22 \%$ of Hepatitis C virus (HCV) patients [117]. Thus hyperglycemic/hyperlipidemic conditions in obesity could favor hepatic hyperplasia development that can acquire malignant traits in the absence of cirrhosis and transform into HCC (Figure 3B). HCC can also arise de novo due to extensive DNA damage and mutations occurring as a result of chronic oxidative stress. Therefore, clinical surveillance of the liver in obese patients is recommended even in the absence of fibrosis/cirrhosis, in order to successfully prevent hepatic malignancy.

\section{A) Canonical model}

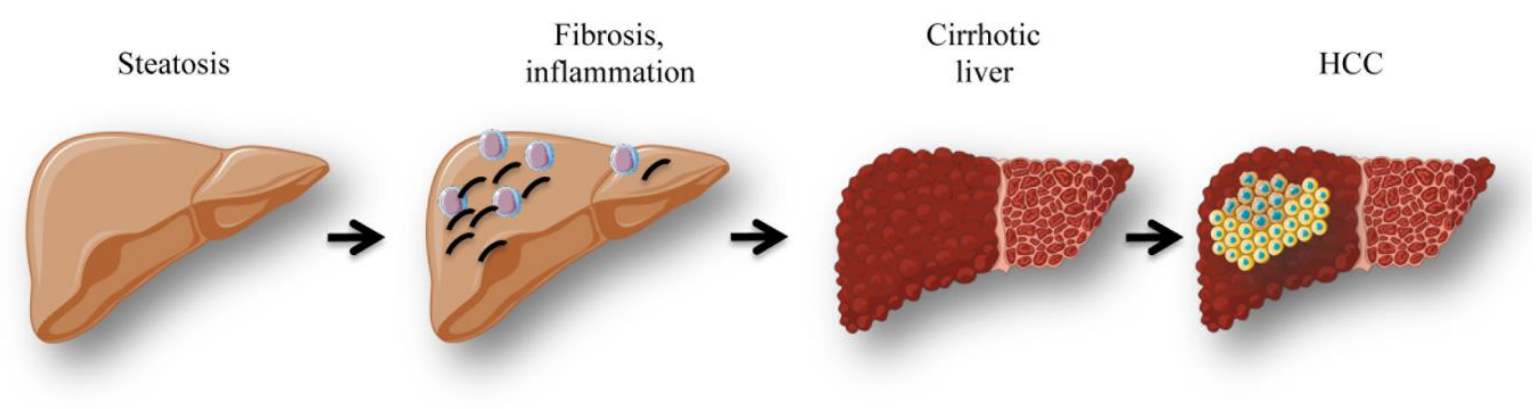

B) Alternative models

Steatosis

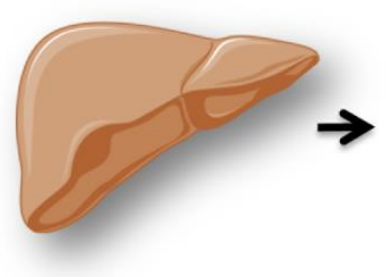

Steatosis
Fibrosis, inflammation
HCC in non-cirrhotic liver

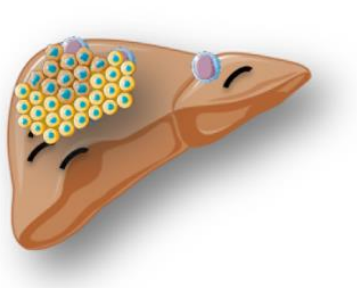

HCC in non fibrotic, non-cirrhotic liver
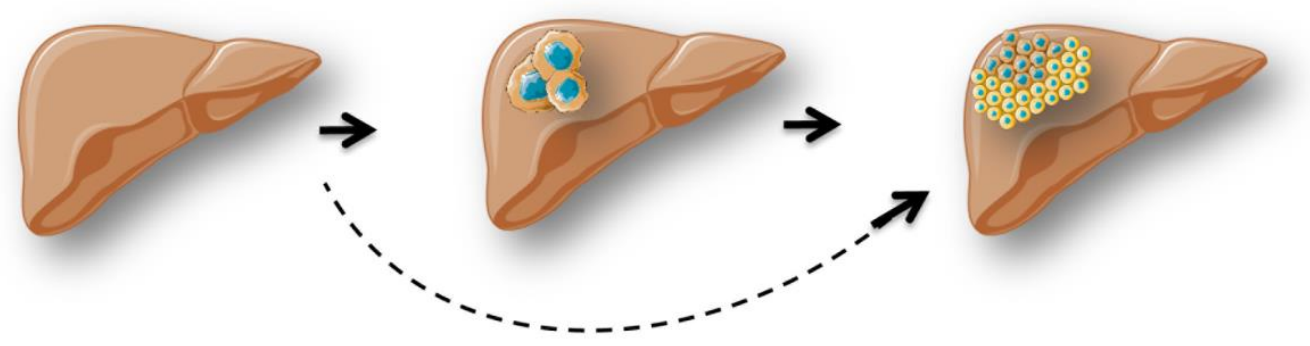

FIGURE 3: Different models of hepatocellular carcinoma development. The canonical model of hepatocellular carcinoma (HCC) development (A) stipulates that patients with hepatic steatosis further develop inflammation / immune cell infiltration and fibrosis. Later on, excessive fibrosis and inflammation can lead to cirrhosis development and HCC. However, alternative models of hepatocarcinogenesis (B) have been observed in obese / GSDI patients. Indeed, steatotic patients, who do not present cirrhosis, can also develop HCC de novo, since fatty livers are favorable for carcinogenesis. Moreover, in GSDI patients, HCC can develop in non-fibrotic, non-cirrhotic liver. These tumors arise from the transformation of hepatocellular adenoma (HCA) to HCC. 
GSDI patients also present an increased risk of hepatic tumor development. Indeed, around $50 \%$ of young adult patients present at least one hepatocellular adenoma (HCA) [118]. There is a high risk (about 10\%) of transformation of HCA into HCC, and this rate is significantly higher in GSDI patients compared to non-GSDI patients. As opposed to HCC patients in the general population, hepatic fibrosis/cirrhosis is absent in GSDI, and therefore de novo formation of $\mathrm{HCC}$ has never been reported. Indeed, all HCC probably arise from a malignant transformation of HCA into HCC (Figure 3B). Thus, malignancy development in GSDI is a very particular linear process, characterized by hepatic steatosis installation, followed by HCA formation, which can later transform into HCC. The exact mechanisms behind this elevated tumor incidence in GSDI are not fully understood. However, the metabolic context in the liver of GSDI patients and animal models could provide a favorable environment for tumorigenesis [26]. As mentioned before, increased glycolysis and subsequent lactate production, elevated lipogenesis and PPP are just some of the metabolic alterations observed in GSDI livers. These metabolic traits are associated to the Warburg effect, a metabolic process infamously affiliated with cancer. Thus in GSDI, the liver itself is characterized by a cancer-like metabolism, potentially facilitating tumor formation and progression.

\section{FINAL REMARKS}

\section{Metabolism and regeneration}

As discussed above, the effects of glycemic imbalance are mostly studied in the central nervous system, the pancreas, in retinopathies and nephropathies, yet, hepatic damage assessment is often overlooked. This is in part due to the exceptional plasticity of the liver and the extraordinary detoxification and regeneration mechanisms that it possesses. Indeed, this organ is capable of efficient regeneration after resection. This process has fascinated mankind since the beginning of medical research, and it has been extensively studied. Some of the facets of liver regeneration remain unknown; however, it has been shown that metabolic aspects are highly important.

Indeed, liver regeneration after partial resection depicts perfectly the plasticity of the metabolism of the liver and how metabolic switches can be crucial in pathophysiology. For example, transient hepatic steatosis appearing right after resection has been reported and described as indispensable for proper regeneration and proliferation of the liver, in order for the hepatocytes to repopulate the liver [119]. In contrast, under these conditions glucose homeostasis is understandably disturbed, since the regenerating liver cannot completely assure the role of the main glucose-producing organ. This depends on the extent to which the organ is resected, the physiopathological condition of the remaining liver, as well as the overall health state of the patient. Thus, regeneration of the liver can lead to hypoglycemia, as often confirmed in many rodent models and patients with partial hepatectomy [120]. Interestingly, supplementing the liver with glucose during this phase can have a negative impact on regeneration [120, 121]. Given that hypoglycemia is thought to induce lipolysis in peripheral organs, facilitating the induction of transient hepatic steatosis needed for regeneration, this outcome is expected, yet it renders post-operative patient care complex. Moreover, hepatic ischemic episodes have been shown to alter glucose metabolism in the liver by switching from oxidative phosphorylation to a more proliferativecompatible metabolism, characterized by activation of glycolysis (the Warburg effect).

\section{Antioxidants against hyperglycemic damages and hepatic tumors}

Since hyperglycemia is strongly associated with oxidative stress, the use of antioxidants in diabetes or in prevention or curative strategies for HCC constituted tempting approaches of treatment. Interestingly, various antioxidant agents such as metformin, Nfr2 agonists, Vitamin C and $\mathrm{E}$, resveratrol, as well as different plant extracts have been used in HCC patients and patients at risk of HCC. The outcomes in these strategies varied greatly among the studies and were described as both pro- and anti-oncogenic [122, 123]. While increased ROS in the cell can be responsible for serious alterations of the DNA and other cell components, it is noteworthy that these molecules are important signaling agents, physiologically needed for the activation of certain defenses in the cell under pre-pathological conditions. Thus it seems important to emphasize that preventing this signalization with antioxidants could be harmful, rather than beneficial. As antioxidants are widely popular in the general population and not only in scientific circles, several misconceptions have been previously highlighted [124]. Indeed, the quantity, the type of antioxidants and the duration of the treatment may have an enormous impact on the outcome for the patient.

While many studies depict the effects of antioxidants in diabetic/obese patients, assessment of the effects of these drugs in GSDI patients is nearly impossible to perform, firstly because of the small number of patient cohorts, but also due to the various treatments that these patients receive in parallel, such as hypolipidemic and hypouricemic agents. However, a study in L.G6pc-/- mice using the antioxidant Tempol showed that while this treatment managed to increase the hepatic expression of Catalase and GPx1, it did not have an impact on carcinogenesis [27].

Last, studies have shown that under some circumstances cancer cells can also be more sensitive to oxidative stress than the surrounding healthy cells [125]. Therefore, inducing oxidative stress in tumor cells is also an attractive strategy to combat tumor progression [126]. To conclude, the redox levels in cancer and the surrounding healthy tissue can vary greatly and one unique approach is not applicable in all patients.

\section{ACKNOWLEDGMENTS}

We would like to thank all past and current colleagues in the laboratory who contributed to the work reviewed. We would particularly like to thank Tania Jauslin (University of Geneva) for her kind contribution to the manuscript. 


\section{CONFLICT OF INTEREST}

The authors declare no conflict of interest.

\section{COPYRIGHT}

(c) 2019 Gjorgjieva et al. This is an open-access article released under the terms of the Creative Commons Attribution (CC BY) license, which allows the unrestricted use,

\section{REFERENCES}

1. Soty M, Gautier-Stein A, Rajas F, and Mithieux G (2017). Gut-Brain Glucose Signaling in Energy Homeostasis. Cell Metab 25(6): 12311242. doi: 10.1016/j.cmet.2017.04.032

2. Gerich JE, Meyer C, Woerle HJ, and Stumvoll M (2001). Renal gluconeogenesis: its importance in human glucose homeostasis. Diabetes Care 24(2): 382-391. doi: 10.2337/diacare.24.2.382

3. Meyer C, Dostou JM, Welle SL, and Gerich JE (2002). Role of human liver, kidney, and skeletal muscle in postprandial glucose homeostasis. Am J Physiol Endocrinol Metab 282(2): E419-427. doi: 10.1152/ajpendo.00032.2001

4. Pillot B, Soty M, Gautier-Stein A, Zitoun C, and Mithieux G (2009). Protein feeding promotes redistribution of endogenous glucose production to the kidney and potentiates its suppression by insulin. Endocrinology 150(2): 616-624. doi: 10.1210/en.2008-0601

5. Mithieux G, Bady I, Gautier A, Croset M, Rajas F, and Zitoun C (2004). Induction of control genes in intestinal gluconeogenesis is sequential during fasting and maximal in diabetes. Am J Physiol Endocrinol Metab 286(3): E370-375. doi: 10.1152/ajpendo.00299.2003

6. Penhoat A, Fayard L, Stefanutti A, Mithieux G, and Rajas F (2014). Intestinal gluconeogenesis is crucial to maintain a physiological fasting glycemia in the absence of hepatic glucose production in mice. Metabolism 63(1): 104-111. doi: 10.1016/j.metabol.2013.09.005

7. Wajchenberg BL (2007). $\beta$-Cell Failure in Diabetes and Preservation by Clinical Treatment. Endocr Rev 28(2): 187-218. doi: 10.1210/10.1210/er.2006-0038

8. Weir GC, and Bonner-Weir S (2004). Five Stages of Evolving BetaCell Dysfunction During Progression to Diabetes. Diabetes 53(Supplement 3): S16-S21. doi: 10.2337/diabetes.53.suppl_3.S16

9. Chen L, Magliano DJ, and Zimmet PZ (2012). The worldwide epidemiology of type 2 diabetes mellitus--present and future perspectives. Nat Rev Endocrinol 8(4): 228-236. doi: 10.1038/nrendo.2011.183

10. Lin HV, and Accili D (2011). Hormonal regulation of hepatic glucose production in health and disease. Cell Metab 14(1): 9-19. doi: 10.1016/j.cmet.2011.06.003

11. Hazlehurst JM, Woods C, Marjot T, Cobbold JF, and Tomlinson JW (2016). Non-alcoholic fatty liver disease and diabetes. Metabolism 65(8): 1096-1108. doi: 10.1016/j.metabol.2016.01.001

12. Williamson RM, Price JF, Glancy S, Perry E, Nee LD, Hayes PC, Frier BM, Van Look LAF, Johnston GI, Reynolds RM, Strachan MWJ, and Edinburgh Type 2 Diabetes Study Investigators (2011). Prevalence of and risk factors for hepatic steatosis and nonalcoholic Fatty liver disease in people with type 2 diabetes: the Edinburgh Type 2 Diabetes Study. Diabetes Care 34(5): 1139-1144. doi: 10.2337/dc10-2229

13. Watanabe $H$, Inaba $Y$, Kimura K, Matsumoto $M$, Kaneko S, Kasuga $\mathrm{M}$, and Inoue $\mathrm{H}$ (2018). Sirt2 facilitates hepatic glucose uptake by deacetylating glucokinase regulatory protein. Nat Commun 9(1): 30 doi: 10.1038/s41467-017-02537-6

14. Schwarz J-M, Linfoot P, Dare D, and Aghajanian K (2003). Hepatic de novo lipogenesis in normoinsulinemic and hyperinsulinemic sub- distribution, and reproduction in any medium, provided the original author and source are acknowledged.

Please cite this article as: Monika Gjorgjieva, Gilles Mithieux and Fabienne Rajas (2019). Hepatic stress associated with pathologies characterized by disturbed glucose production. Cell Stress 3(3): 86-99. doi: 10.15698/cst2019.03.179

jects consuming high-fat, low-carbohydrate and low-fat, highcarbohydrate isoenergetic diets. Am J Clin Nutr 77(1): 43-50. doi: 10.1093/ajcn/77.1.43

15. Abdul-Wahed A, Guilmeau S, and Postic C (2017). Sweet Sixteenth for ChREBP: Established Roles and Future Goals. Cell Metab 26(2): 324-341. doi: 10.1016/j.cmet.2017.07.004

16. Browning JD, and Horton JD (2004). Molecular mediators of hepatic steatosis and liver injury. J Clin Invest 114(2): 147-152. doi $10.1172 / \mathrm{JCl} 200422422$

17. Wang $Y$, Viscarra J, Kim S-J, and Sul HS (2015). Transcriptional regulation of hepatic lipogenesis. Nat Rev Mol Cell Biol 16(11): 678689. doi: $10.1038 / \mathrm{nrm} 4074$

18. Postic C, and Girard J (2008). Contribution of de novo fatty acid synthesis to hepatic steatosis and insulin resistance: lessons from genetically engineered mice. J Clin Invest 118(3): 829-838. doi: $10.1172 / \mathrm{JCl} 34275$

19. Kishnani PS, Austin SL, Abdenur JE, Arn P, Bali DS, Boney A, Chung WK, Dagli Al, Dale D, Koeberl D, Somers MJ, Wechsler SB, Weinstein DA, Wolfsdorf JI, Watson MS, and American College of Medical Genetics and Genomics (2014). Diagnosis and management of glycogen storage disease type I: a practice guideline of the American College of Medical Genetics and Genomics. Genet Med Off J Am Coll Med Genet 16(11): e1. doi: 10.1038/gim.2014.128

20. Bruni N, Rajas F, Montano S, Chevalier-Porst F, Maire I, and Mithieux $G$ (1999). Enzymatic characterization of four new mutations in the glucose- 6 phosphatase (G6PC) gene which cause glycogen storage disease type 1a. Ann Hum Genet 63(Pt 2): 141-146. doi: 10.1046/j.1469-1809.1999.6320141.x

21. Chevalier-Porst F, Bozon D, Bonardot AM, Bruni N, Mithieux G, Mathieu M, and Maire I (1996). Mutation analysis in 24 French patients with glycogen storage disease type 1a. J Med Genet 33(5): 358360. doi: 10.1136/jmg.33.5.358

22. Bandsma RHJ, Smit GPA, and Kuipers F (2014). Disturbed lipid metabolism in glycogen storage disease type 1. Eur J Pediatr 161(1): S65-S69. doi: 10.1007/BF02679998

23. Sun B, Li S, Yang L, Damodaran T, Desai D, Diehl AM, Alzate O, and Koeberl DD (2009). Activation of glycolysis and apoptosis in glycogen storage disease type la. Mol Genet Metab 97(4): 267-271. doi: 10.1016/j.ymgme.2009.04.003

24. Bandsma RHJ, Prinsen $B H$, de Sain-van der Velden $M$, Rake J-P, Boer T, Smit GPA, Reijngoud D-J, and Kuipers F (2008). Increased de novo Lipogenesis and Delayed Conversion of Large VLDL into Intermediate Density Lipoprotein Particles Contribute to Hyperlipidemia in Glycogen Storage Disease Type 1a. Pediatr Res 63(6): 702-707. doi: 10.1203/PDR.0b013e31816c9013

25. Rajas F, Jourdan-Pineau $H$, Stefanutti A, Mrad EA, Iynedjian PB, and Mithieux $G$ (2007). Immunocytochemical localization of glucose 6 phosphatase and cytosolic phosphoenolpyruvate carboxykinase in gluconeogenic tissues reveals unsuspected metabolic zonation. Histochem Cell Biol 127(5): 555-565. doi: 10.1007/s00418-006-0263-5 
26. Gjorgjieva M, Oosterveer MH, Mithieux G, and Rajas F (2016). Mechanisms by Which Metabolic Reprogramming in GSD1 Liver Generates a Favorable Tumorigenic Environment. J Inborn Errors Metab Screen 4: 2326409816679429. doi: 10.1177/2326409816679429

27. Gjorgjieva M, Calderaro J, Monteillet L, Silva M, Raffin M, Brevet $M$ Romestaing C, Roussel D, Zucman-Rossi J, Mithieux G, and Rajas F (2018). Dietary exacerbation of metabolic stress leads to accelerated hepatic carcinogenesis in glycogen storage disease type la. J Hepatol 69(5): 1074-1087. doi: 10.1016/j.jhep.2018.07.017

28. Cho J-H, Kim G-Y, Mansfield BC, and Chou JY (2018). Hepatic glucose-6-phosphatase- $\alpha$ deficiency leads to metabolic reprogramming in glycogen storage disease type la. Biochem Biophys Res Commun 498(4): 925-931. doi: 10.1016/j.bbrc.2018.03.083

29. Rajas F, Labrune $P$, and Mithieux G (2013). Glycogen storage disease type 1 and diabetes: learning by comparing and contrasting the two disorders. Diabetes Metab 39(5): 377-387. doi: 10.1016/j.diabet.2013.03.002

30. Gargouri M, Magné C, and El Feki A (2016). Hyperglycemia, oxidative stress, liver damage and dysfunction in alloxan-induced diabetic rat are prevented by Spirulina supplementation. Nutr Res 36(11): 1255-1268. doi: 10.1016/j.nutres.2016.09.011

31. Manna P, Das J, Ghosh J, and Sil PC (2010). Contribution of type 1 diabetes to rat liver dysfunction and cellular damage via activation of NOS, PARP, IkappaBalpha/NF-kappaB, MAPKs, and mitochondriadependent pathways: Prophylactic role of arjunolic acid. Free Radic Biol Med 48(11): 1465-1484. doi: 10.1016/j.freeradbiomed.2010.02.025

32. Ashraf NU, and Sheikh TA (2015). Endoplasmic reticulum stress and Oxidative stress in the pathogenesis of Non-alcoholic fatty liver disease. Free Radic Res 49(12): 1405-1418. doi: 10.3109/10715762.2015.1078461

33. Spahis S, Delvin E, Borys J-M, and Levy E (2017). Oxidative Stress as a Critical Factor in Nonalcoholic Fatty Liver Disease Pathogenesis. Antioxid Redox Signal 26(10): 519-541. doi: 10.1089/ars.2016.6776

34. Forbes JM, and Cooper ME (2013). Mechanisms of diabetic complications. Physiol Rev 93(1): 137-188. doi: 10.1152/physrev.00045.2011

35. Chen X-J, Wu W-J, Zhou Q, Jie J-P, Chen X, Wang F, and Gong X-H (2018). Advanced glycation end-products induce oxidative stress through the Sirt1/Nrf2 axis by interacting with the receptor of AGEs under diabetic conditions. J Cell Biochem 120(2): 2159-2170. doi: $10.1002 / \mathrm{jcb} .27524$

36. Filomeni G, De Zio D, and Cecconi F (2015). Oxidative stress and autophagy: the clash between damage and metabolic needs. Cell Death Differ 22(3): 377-388. doi: 10.1038/cdd.2014.150

37. Satapati S, Kucejova B, Duarte JAG, Fletcher JA, Reynolds L, Sunny NE, He T, Nair LA, Livingston K, Fu X, Merritt ME, Sherry AD, Malloy CR, Shelton JM, Lambert J, Parks EJ, Corbin I, Magnuson MA, Browning JD, and Burgess SC (2015). Mitochondrial metabolism mediates oxidative stress and inflammation in fatty liver. J Clin Invest 125(12): 4447-4462. doi: $10.1172 / \mathrm{JCl} 82204$

38. Sunny NE, Bril F, and Cusi K (2017). Mitochondrial Adaptation in Nonalcoholic Fatty Liver Disease: Novel Mechanisms and Treatment Strategies. Trends Endocrinol Metab TEM 28(4): 250-260. doi: 10.1016/j.tem.2016.11.006

39. Lorenzi M (2007). The polyol pathway as a mechanism for diabetic retinopathy: attractive, elusive, and resilient. Exp Diabetes Res 2007: 61038. doi: 10.1155/2007/61038

40. Rolo AP, and Palmeira CM (2006). Diabetes and mitochondrial function: role of hyperglycemia and oxidative stress. Toxicol Appl Pharmacol 212(2): 167-178. doi: 10.1016/j.taap.2006.01.003
41. Vlassara $\mathrm{H}$, and Striker GE (2011). AGE restriction in diabetes mellitus: a paradigm shift. Nat Rev Endocrinol 7(9): 526-539. doi: 10.1038/nrendo.2011.74

42. Horiuchi S (2002). The liver is the main site for metabolism of circulating advanced glycation end products. J Hepatol 36(1): 123-125. doi: 10.1016/s0168-8278(01)00293-8

43. Ahmed N (2005). Advanced glycation endproducts--role in pathology of diabetic complications. Diabetes Res Clin Pract 67(1): 3-21. doi: 10.1016/j.diabres.2004.09.004

44. Buse MG (2006). Hexosamines, insulin resistance and the complications of diabetes: current status. Am J Physiol Endocrinol Metab 290(1): E1-E8. doi: 10.1152/ajpendo.00329.2005

45. Schleicher ED, and Weigert C (2000). Role of the hexosamine biosynthetic pathway in diabetic nephropathy. Kidney Int Suppl 77: S1318. doi: 10.1046/j.1523-1755.2000.07703.x

46. Murphy MP (2009). How mitochondria produce reactive oxygen species. Biochem J 417(Pt 1): 1-13. doi: 10.1042/BJ20081386

47. Sindhu RK, Koo J-R, Roberts CK, and Vaziri ND (2004). Dysregulation of hepatic superoxide dismutase, catalase and glutathione peroxidase in diabetes: response to insulin and antioxidant therapies. Clin Exp Hypertens 26(1): 43-53. doi: 10.1081/ceh-120027330

48. Lao-ong T, Chatuphonprasert W, Nemoto N, and Jarukamjorn K (2012). Alteration of hepatic glutathione peroxidase and superoxide dismutase expression in streptozotocin-induced diabetic mice by berberine. Pharm Biol 50(8): 1007-1012. doi: 10.3109/13880209.2012.655377

49. Fedorova M, Bollineni RC, and Hoffmann R (2014). Protein carbonylation as a major hallmark of oxidative damage: update of analytical strategies. Mass Spectrom Rev 33(2): 79-97. doi: 10.1002/mas.21381

50. Jena NR (2012). DNA damage by reactive species: Mechanisms, mutation and repair. J Biosci 37(3): 503-517. doi: 10.1007/s12038012-9218-2

51. Lee SC, and Chan JC (2015). Evidence for DNA Damage as a Biological Link Between Diabetes and Cancer. Chin Med J 128(11): 15431548. doi: 10.4103/0366-6999.157693

52. Schmid Al, Szendroedi J, Chmelik M, Krššák M, Moser E, and Roden M (2011). Liver ATP Synthesis Is Lower and Relates to Insulin Sensitivity in Patients With Type 2 Diabetes. Diabetes Care 34(2): 448-453. doi: $10.2337 /$ dc10-1076

53. Jain M, Ngoy S, Sheth SA, Swanson RA, Rhee EP, Liao R, Clish CB, Mootha VK, and Nilsson $R$ (2014). A systematic survey of lipids across mouse tissues. Am J Physiol-Endocrinol Metab 306(8): E854-E868. doi: 10.1152/ajpendo.00371.2013

54. Greenberg AS, Egan JJ, Wek SA, Garty NB, Blanchette-Mackie EJ, and Londos $C$ (1991). Perilipin, a major hormonally regulated adipocyte-specific phosphoprotein associated with the periphery of lipid storage droplets. J Biol Chem 266(17): 11341-11346. 2040638

55. Gluchowski NL, Becuwe M, Walther TC, and Farese RV (2017) Lipid droplets and liver disease: from basic biology to clinical implications. Nat Rev Gastroenterol Hepatol 14(6): 343-355. doi: 10.1038/nrgastro.2017.32

56. Yamaguchi K, Yang L, McCall S, Huang J, Yu XX, Pandey SK, Bhanot S, Monia BP, Li Y-X, and Diehl AM (2007). Inhibiting triglyceride synthesis improves hepatic steatosis but exacerbates liver damage and fibrosis in obese mice with nonalcoholic steatohepatitis. Hepatol Baltim Md 45(6): 1366-1374. doi: 10.1002/hep.21655

57. Alkhouri N, Dixon LJ, and Feldstein AE (2009). Lipotoxicity in Nonalcoholic Fatty Liver Disease: Not All Lipids Are Created Equal. Expert Rev Gastroenterol Hepatol 3(4): 445-451. doi: 10.1586/egh.09.32 
58. Pelusi S, and Valenti L (2018). Hepatic fat as clinical outcome and therapeutic target for nonalcoholic fatty liver disease. Liver Int. doi: 10.1111/liv.13972.

59. Erion DM, and Shulman GI (2010). Diacylglycerol-mediated insulin resistance. Nat Med 16(4): 400-402. doi: 10.1038/nm0410-400

60. Galadari S, Rahman A, Pallichankandy S, Galadari A, and Thayyullathil $F$ (2013). Role of ceramide in diabetes mellitus: evidence and mechanisms. Lipids Health Dis 12: 98. doi: 10.1186/1476-511X12-98

61. Weltman MD, Farrell GC, Hall $P$, Ingelman-Sundberg $M$, and Liddle $C$ (1998). Hepatic cytochrome P450 2E1 is increased in patients with nonalcoholic steatohepatitis. Hepatol Baltim Md 27(1): 128-133. doi: 10.1002/hep.510270121

62. Linhart K, Bartsch H, and Seitz HK (2014). The role of reactive oxygen species (ROS) and cytochrome P-450 2E1 in the generation of carcinogenic etheno-DNA adducts. Redox Biol 3: 56-62. doi: 10.1016/j.redox.2014.08.009

63. Fu S, Yang L, Li P, Hofmann O, Dicker L, Hide W, Lin X, Watkins SM, Ivanov A, and Hotamisligil GS (2011). Aberrant lipid metabolism disrupts calcium homeostasis causing liver endoplasmic reticulum stress in obesity. Nature 473(7348): 528-531. doi: 10.1038/nature09968

64. Zhang C, Lu X, Tan Y, Li B, Miao X, Jin L, Shi X, Zhang X, Miao L, Li X, and Cai $L$ (2012). Diabetes-Induced Hepatic Pathogenic Damage, Inflammation, Oxidative Stress, and Insulin Resistance Was Exacerbated in Zinc Deficient Mouse Model. PLoS ONE 7(12). doi: 10.1371/journal.pone.0049257

65. Han J, and Kaufman RJ (2016). The role of ER stress in lipid metabolism and lipotoxicity. J Lipid Res 57(8): 1329-1338. doi: 10.1194/jlr.R067595

66. Ferré $P$, and Foufelle $F$ (2010). Hepatic steatosis: a role for de novo lipogenesis and the transcription factor SREBP-1c. Diabetes Obes Metab 12 Suppl 2: 83-92. doi: 10.1111/j.1463-1326.2010.01275.x

67. Cichoż-Lach H, and Michalak A (2014). Oxidative stress as a crucial factor in liver diseases. World J Gastroenterol 20(25): 8082-8091. doi: 10.3748/wjg.v20.i25.8082

68. Mohamed J, Nazratun Nafizah AH, Zariyantey AH, and Budin SB (2016). Mechanisms of Diabetes-Induced Liver Damage. Sultan Qaboos Univ Med J 16(2): e132-e141. doi: 10.18295/squmj.2016.16.02.002

69. Leclercq IA, Morais ADS, Schroyen B, Hul NV, and Geerts A (2007). Insulin resistance in hepatocytes and sinusoidal liver cells: Mechanisms and consequences. J Hepatol 47(1): 142-156. doi: 10.1016/j.jhep.2007.04.002

70. Banerjee M, and Saxena M (2012). Interleukin-1 (IL-1) family of cytokines: role in type 2 diabetes. Clin Chim Acta Int J Clin Chem 413(15-16): 1163-1170. doi: 10.1016/j.cca.2012.03.021

71. Ingaramo PI, Ronco MT, Francés DEA, Monti JA, Pisani GB, Ceballos MP, Galleano M, Carrillo MC, and Carnovale CE (2011). Tumor necrosis factor alpha pathways develops liver apoptosis in type 1 diabetes mellitus. Mol Immunol 48(12-13): 1397-1407. doi: 10.1016/j.molimm.2011.03.015

72. Francés DE, Ingaramo PI, Ronco MT, and Carnovale CE (2013). Diabetes, an inflammatory process: Oxidative Stress and TNF-alpha involved in hepatic complication. J Biomed Sci Eng 06(06): 645. doi: 10.4236/jbise.2013.66079

73. Klover PJ, Zimmers TA, Koniaris LG, and Mooney RA (2003). Chronic exposure to interleukin- 6 causes hepatic insulin resistance in mice. Diabetes 52(11): 2784-2789. doi: 10.2337/diabetes.52.11.2784

74. Schmidt-Arras D, and Rose-John S (2016). IL-6 pathway in the liver: From physiopathology to therapy. J Hepatol 64(6): 1403-1415. doi:

\subsection{6/j.jhep.2016.02.004}

75. Wunderlich FT, Ströhle P, Könner AC, Gruber S, Tovar S, Brönneke HS, Juntti-Berggren L, Li L-S, van Rooijen N, Libert C, Berggren P-O, and Brüning JC (2010). Interleukin-6 signaling in liver-parenchymal cells suppresses hepatic inflammation and improves systemic insulin action. Cell Metab 12(3): 237-249. doi: 10.1016/j.cmet.2010.06.011

76. Gao B, and Tsukamoto H (2016). Inflammation in Alcoholic and Nonalcoholic Fatty Liver Disease: Friend or Foe? Gastroenterology 150(8): 1704-1709. doi: 10.1053/j.gastro.2016.01.025

77. Gadd VL, Skoien R, Powell EE, Fagan KJ, Winterford C, Horsfall L, Irvine K, and Clouston AD (2014). The portal inflammatory infiltrate and ductular reaction in human nonalcoholic fatty liver disease. Hepatol Baltim Md 59(4): 1393-1405. doi: 10.1002/hep.26937

78. Ma C, Kesarwala AH, Eggert T, Medina-Echeverz J, Kleiner DE, Jin $P$, Stroncek DF, Terabe M, Kapoor V, ElGindi M, Han M, Thornton AM, Zhang $\mathrm{H}$, Egger M, Luo J, Felsher DW, McVicar DW, Weber A, Heikenwalder M, and Greten TF (2016). NAFLD causes selective CD4(+) T lymphocyte loss and promotes hepatocarcinogenesis. Nature 531(7593): 253-257. doi: 10.1038/nature16969

79. Archuleta TL, Lemieux AM, Saengsirisuwan V, Teachey MK, Lindborg KA, Kim JS, and Henriksen EJ (2009). Oxidant stress-induced loss of IRS-1 and IRS-2 proteins in rat skeletal muscle: role of p38 MAPK. Free Radic Biol Med 47(10): 1486-1493. doi: 10.1016/j.freeradbiomed.2009.08.014

80. Rossi A, Ruoppolo M, Formisano P, Villani G, Albano L, Gallo G, Crisci D, Moccia A, Parenti G, Strisciuglio P, and Melis D (2018). Insulin-resistance in glycogen storage disease type la: linking carbohydrates and mitochondria? J Inherit Metab Dis. doi: 10.1007/s10545018-0149-4

81. Lei KJ, Chen H, Pan CJ, Ward JM, Mosinger B, Lee EJ, Westphal H, Mansfield BC, and Chou JY (1996). Glucose-6-phosphatase dependent substrate transport in the glycogen storage disease type-1a mouse. Nat Genet 13(2): 203-209. doi: 10.1038/ng0696-203

82. Mutel E, Abdul-Wahed A, Ramamonjisoa N, Stefanutti A, Houberdon I, Cavassila S, Pilleul F, Beuf O, Gautier-Stein A, Penhoat A, Mithieux G, and Rajas $F$ (2011). Targeted deletion of liver glucose- 6 phosphatase mimics glycogen storage disease type 1a including development of multiple adenomas. J Hepatol 54(3): 529-537. doi: 10.1016/j.jhep.2010.08.014

83. Mutel $E$, Gautier-Stein A, Abdul-Wahed A, Amigó-Correig $M$ Zitoun C, Stefanutti A, Houberdon I, Tourette J-A, Mithieux G, and Rajas $F$ (2011). Control of Blood Glucose in the Absence of Hepatic Glucose Production During Prolonged Fasting in Mice. Diabetes 60(12): 3121-3131. doi: 10.2337/db11-0571

84. Farah BL, Sinha RA, Wu Y, Singh BK, Lim A, Hirayama M, Landau DJ, Bay BH, Koeberl DD, and Yen PM (2017). Hepatic mitochondrial dysfunction is a feature of Glycogen Storage Disease Type la (GSDla). Sci Rep 7: 44408. doi: 10.1038/srep44408

85. Cho J-H, Kim G-Y, Mansfield BC, and Chou JY (2018). Sirtuin signaling controls mitochondrial function in glycogen storage disease type la. J Inherit Metab Dis 1-10. doi: 10.1007/s10545-018-0192-1

86. Wittenstein B, Klein M, Finckh B, Ullrich $K$, and Kohlschütter A (2002). Plasma antioxidants in pediatric patients with glycogen storage disease, diabetes mellitus, and hypercholesterolemia. Free Radic Biol Med 33(1): 103-110. doi: 10.1016/s0891-5849(02)00863-8

87. Kalkan Ucar S, Coker M, Sözmen E, Goksen Simsek D, and Darcan S (2009). A monocentric pilot study of an antioxidative defense and hsCRP in pediatric patients with glycogen storage disease type IA and III. Nutr Metab Cardiovasc Dis 19(6): 383-390. doi: 10.1016/j.numecd.2008.09.005

88. Koren E, Lipkin J, Klar A, Hershkovitz E, Ginsburg I, and Kohen R 
(2009). Total oxidant-scavenging capacities of plasma from glycogen storage disease type la patients as measured by cyclic voltammetry, FRAP and luminescence techniques. J Inherit Metab Dis 32(5): 651. doi: $10.1007 /$ s10545-009-1242-5

89. Sautin YY, and Johnson RJ (2008). URIC ACID: THE OXIDANTANTIOXIDANT PARADOX. Nucleosides Nucleotides Nucleic Acids 27(6): 608-619. doi: 10.1080/15257770802138558

90. Abdul-Wahed A, Gautier-Stein A, Casteras S, Soty M, Roussel D, Romestaing C, Guillou H, Tourette J-A, Pleche N, Zitoun C, Gri B, Sardella A, Rajas F, and Mithieux $G$ (2014). A link between hepatic glucose production and peripheral energy metabolism via hepatokines. Mol Metab 3(5): 531-543. doi: 10.1016/j.molmet.2014.05.005

91. Monteillet $L$, Gjorgjieva $M$, Silva $M$, Verzieux V, Imikirene $L$, Duchampt A, Guillou H, Mithieux G, and Rajas F (2018). Intracellular lipids are an independent cause of liver injury and chronic kidney disease in non alcoholic fatty liver disease-like context. Mol Metab 16: 100-115.doi: 10.1016/j.molmet.2018.07.006

92. Farah BL, Landau DJ, Sinha RA, Brooks ED, Wu Y, Fung SYS, Tanaka T, Hirayama M, Bay B-H, Koeberl DD, and Yen PM (2016). Induction of autophagy improves hepatic lipid metabolism in glucose-6phosphatase deficiency. J Hepatol 64(2): 370-379. doi: 10.1016/j.jhep.2015.10.008

93. Cho J-H, Kim G-Y, Pan C-J, Anduaga J, Choi E-J, Mansfield BC, and Chou JY (2017). Downregulation of SIRT1 signaling underlies hepatic autophagy impairment in glycogen storage disease type la. PLOS Genet 13(5): e1006819. doi: 10.1371/journal.pgen.1006819

94. Mathew R, Karantza-Wadsworth V, and White E (2007). Role of autophagy in cancer. Nat Rev Cancer 7(12): 961-967. doi: $10.1038 / \mathrm{nrc} 2254$

95. Kroemer G, Mariño G, and Levine B (2010). Autophagy and the integrated stress response. Mol Cell 40(2): 280-293. doi: 10.1016/j.molcel.2010.09.023

96. Namkoong S, Cho C-S, Semple I, and Lee JH (2018). Autophagy Dysregulation and Obesity-Associated Pathologies. Mol Cells 41(1): 310. doi: $10.14348 /$ molcells.2018.2213

97. Qian $Q$, Zhang Z, Orwig A, Chen S, Ding W-X, Xu Y, Kunz RC, Lind NRL, Stamler JS, and Yang L (2018). S-Nitrosoglutathione Reductase Dysfunction Contributes to Obesity-Associated Hepatic Insulin Resistance via Regulating Autophagy. Diabetes 67(2): 193-207. doi: $10.2337 / \mathrm{db} 17-0223$

98. Lavallard VJ, and Gual P (2014). Autophagy and non-alcoholic fatty liver disease. BioMed Res Int 2014: 120179. doi: 10.1155/2014/120179

99. Mei S, Ni H-M, Manley S, Bockus A, Kassel KM, Luyendyk JP, Copple BL, and Ding W-X (2011). Differential Roles of Unsaturated and Saturated Fatty Acids on Autophagy and Apoptosis in Hepatocytes. J Pharmacol Exp Ther 339(2): 487-498. doi: 10.1124/jpet.111.184341

100. Kim SY, Weinstein DA, Starost MF, Mansfield BC, and Chou JY (2008). Necrotic foci, elevated chemokines and infiltrating neutrophils in the liver of glycogen storage disease type la. J Hepatol 48(3): 479485. doi: 10.1016/j.jhep.2007.11.014

101. Blendea MC, Thompson MJ, and Malkani S (2010). Diabetes and Chronic Liver Disease: Etiology and Pitfalls in Monitoring. Clin Diabetes 28(4): 139-144. doi: 10.2337/diaclin.28.4.139

102. Fujita K, Iwama H, Miyoshi H, Tani J, Oura K, Tadokoro T, Sakamoto T, Nomura T, Morishita A, Yoneyama H, and Masaki T (2016). Diabetes mellitus and metformin in hepatocellular carcinoma. World J Gastroenterol 22(27): 6100-6113. doi: 10.3748/wjg.v22.i27.6100

103. Wang P, Kang D, Cao W, Wang Y, and Liu Z (2012). Diabetes mellitus and risk of hepatocellular carcinoma: a systematic review and meta-analysis. Diabetes Metab Res Rev 28(2): 109-122. doi: 10.1002/dmrr.1291

104. Yang JD, Mohamed HA, Cvinar JL, Gores GJ, Roberts LR, and Kim WR (2016). Diabetes Mellitus Heightens the Risk of Hepatocellular Carcinoma Except in Patients With Hepatitis C Cirrhosis. Am J Gastroenterol 111(11): 1573-1580. doi: 10.1038/ajg.2016.330

105. Giovannucci E, Harlan DM, Archer MC, Bergenstal RM, Gapstur SM, Habel LA, Pollak M, Regensteiner JG, and Yee D (2010). Diabetes and cancer: a consensus report. CA Cancer J Clin 60(4): 207-221. doi: 10.3322/caac. 20078

106. Chettouh H, Lequoy $M$, Fartoux L, Vigouroux C, and DesboisMouthon C (2015). Hyperinsulinaemia and insulin signalling in the pathogenesis and the clinical course of hepatocellular carcinoma. Liver Int Off J Int Assoc Study Liver 35(10): 2203-2217. doi: 10.1111/liv.12903

107. Chocarro-Calvo A, García-Martínez JM, Ardila-González S, De la Vieja A, and García-Jiménez C (2013). Glucose-induced $\beta$-catenin acetylation enhances Wnt signaling in cancer. Mol Cell 49(3): 474-486. doi: 10.1016/j.molcel.2012.11.022

108. Bertolani $C$, and Marra $F$ (2008). The role of adipokines in liver fibrosis. Pathophysiol Off J Int Soc Pathophysiol 15(2): 91-101. doi: 10.1016/j.pathophys.2008.05.001

109. Crespo J, Cayón A, Fernández-Gil P, Hernández-Guerra $M$ Mayorga M, Domínguez-Díez A, Fernández-Escalante JC, and PonsRomero $F$ (2001). Gene expression of tumor necrosis factor alpha and TNF-receptors, p55 and p75, in nonalcoholic steatohepatitis patients. Hepatol Baltim Md 34(6): 1158-1163. doi: 10.1053/jhep.2001.29628

110. Friedman SL (2008). Hepatic stellate cells: protean, multifunctional, and enigmatic cells of the liver. Physiol Rev 88(1): 125-172. doi: 10.1152/physrev.00013.2007

111. Park P-H, Sanz-Garcia C, and Nagy LE (2015). Adiponectin as an anti-fibrotic and anti-inflammatory adipokine in the liver. Curr Pathobiol Rep 3(4): 243-252. doi: 10.1007/s40139-015-0094-y

112. Jensen K, and Gluud C (1994). The Mallory body: morphological, clinical and experimental studies (Part 1 of a literature survey). Hepatol Baltim Md 20(4 Pt 1): 1061-1077. 7927209

113. Garcia-Compean D, Jaquez-Quintana JO, Gonzalez-Gonzalez JA, and Maldonado-Garza H (2009). Liver cirrhosis and diabetes: Risk factors, pathophysiology, clinical implications and management. World J Gastroenterol 15(3): 280-288. doi: 10.3748/wjg.15.280

114. Hsieh P-H, Huang J-Y, Nfor ON, Lung C-C, Ho C-C, and Liaw Y-P (2017). Association of type 2 diabetes with liver cirrhosis: a nationwide cohort study. Oncotarget 8(46): 81321-81328. doi: 10.18632/oncotarget.18466

115. Baffy G (2013). Hepatocellular Carcinoma in Non-alcoholic Fatty Liver Disease: Epidemiology, Pathogenesis, and Prevention. J Clin Transl Hepatol 1(2): 131-137. doi: 10.14218/JCTH.2013.00005

116. Calzadilla Bertot L, and Adams LA (2016). The Natural Course of Non-Alcoholic Fatty Liver Disease. Int J Mol Sci 17(5). doi: 10.3390/ijms17050774

117. Sanyal A, Poklepovic A, Moyneur E, and Barghout V (2010). Population-based risk factors and resource utilization for HCC: US perspective. Curr Med Res Opin 26(9): 2183-2191. doi 10.1185/03007995.2010.506375

118. Wang DQ, Fiske LM, Carreras CT, and Weinstein DA (2011). NATURAL HISTORY OF HEPATOCELLULAR ADENOMA FORMATION IN GLYCOGEN STORAGE DISEASE TYPE I. J Pediatr 159(3): 442-446. doi: 10.1016/j.jpeds.2011.02.031

119. Rudnick DA, and Davidson NO (2012). Functional Relationships between Lipid Metabolism and Liver Regeneration. Int J Hepatol 


\section{2: 549241. doi: 10.1155/2012/549241}

120. Huang J, Schriefer AE, Cliften PF, Dietzen D, Kulkarni S, Sing S, Monga SPS, and Rudnick DA (2016). Postponing the Hypoglycemic Response to Partial Hepatectomy Delays Mouse Liver Regeneration. Am J Pathol 186(3): 587-599. doi: 10.1016/j.ajpath.2015.10.027

121. Holeček M (1999). Nutritional modulation of liver regeneration by carbohydrates, lipids, and amino acids: a review. Nutrition 15(10): 784-788. doi: 10.1016/S0899-9007(99)00158-6

122. Miyanishi K, Hoki T, Tanaka S, and Kato J (2015). Prevention of hepatocellular carcinoma: Focusing on antioxidant therapy. World J Hepatol 7(3): 593-599. doi: 10.4254/wjh.v7.i3.593

123. Takaki A, and Yamamoto K (2015). Control of oxidative stress in hepatocellular carcinoma: Helpful or harmful? World J Hepatol 7(7): 968-979. doi: 10.4254/wjh.v7.i7.968

124. Bast A, and Haenen GRMM (2013). Ten misconceptions about antioxidants. Trends Pharmacol Sci 34(8): 430-436. doi: 10.1016/j.tips.2013.05.010

125. Mendelsohn AR, and Larrick JW (2014). Paradoxical effects of antioxidants on cancer. Rejuvenation Res 17(3): 306-311. doi: 10.1089/rej.2014.1577

126. Wang J, and Yi J (2008). Cancer cell killing via ROS: to increase or decrease, that is the question. Cancer Biol Ther 7(12): 1875-1884. doi: $10.4161 /$ cbt.7.12.7067 